\title{
Plasma insulin concentrations in hypoglycaemic dogs with Babesia canis rossi infection
}

\author{
P. Rees, J.P. Schoeman \\ Department of Companion Animal Clinical Studies, Faculty of Veterinary Science, \\ University of Pretoria, Pretoria, Onderstepoort 0110, South Africa \\ With 1 table and 1 figure
}

\begin{abstract}
Hypoglycaemia has been identified as a life-threatening metabolic complication in almost $20 \%$ of severely ill dogs suffering from babesiosis due to Babesia canis rossi infection, and has been correlated with mortality. Hyperinsulinaemia as a result of inappropriate insulin secretion may precipitate hypoglycaemia, and has been suggested as a possible cause of hypoglycaemia in human and murine malaria. This prospective, cross-sectional, observational study, including 94 dogs with naturally occurring virulent babesiosis, sought to identify the presence of inappropriate insulin secretion in hypoglycaemic canine babesiosis.

Pre-treatment jugular blood samples were collected for simultaneous determination of plasma glucose and insulin concentrations. Animals were retrospectively divided into three groups: hypoglycaemic (BG $<3.3 \mathrm{mmol} / \mathrm{L} ; n=16)$, normoglycaemic (BG 3.3-5.5 mmol/L; $n=62$ ), and hyperglycaemic (BG $>5.5 \mathrm{mmol} / \mathrm{L} ; n=16)$. The median insulin concentrations for the hypoglycaemic, normoglycaemic, and hyperglycaemic groups were $10.7 \mathrm{pmol} / \mathrm{L}, 10.7$ $\mathrm{pmol} / \mathrm{L}$, and $21.7 \mathrm{pmol} / \mathrm{L}$, respectively. Statistical analysis revealed no significant difference in insulin concentration between the three groups. Additionally, the median insulin concentration in the hypoglycaemic and normoglycaemic groups was below the detection limit of the assay, suggesting that insulin secretion was appropriately low (i.e. undetectable) in these cases. Only two dogs had inappropriately elevated insulin concentrations. One of these dogs was hypoglycaemic.

We conclude that hyperinsulinaemia is an infrequent cause of hypoglycaemia in virulent canine babesiosis. Other causes of hypoglycaemia, such as increased glucose consumption, depletion of hepatic glycogen stores, and hepatic dysfunction with impaired gluconeogenesis, are speculated to play more important roles in the pathophysiology of hypoglycaemia in canine babesiosis.
\end{abstract}

Keywords: Babesia canis rossi; Malaria; Insulin; Hypoglycaemia

\section{Introduction}

Canine babesiosis is a severe, often life-threatening parasitic disease affecting dogs. Babesia canis rossi, the aetiologic agent of virulent babesiosis in South Africa produces acute disease in susceptible individuals (Jacobson, 2006). The disease is characterized by acute haemolysis and systemic inflammation, and severe complications may arise, including immune-mediated haemolytic anaemia, acute renal failure (Lobetti and Jacobson, 2001), pulmonary oedema (Jacobson, 2006), and pancreatitis (Mohr et al., 2000). Hypoglycaemia was recently identified as a life-threatening metabolic complication in almost $20 \%$ of severely ill dogs suffering from babesiosis due to $B$. canis rossi infection in South Africa (Keller et al., 2004). Hypoglycaemia and hyperlactataemia are correlated with a poor outcome in various human critical illnesses (Bakker et al., 1996) including malaria (White et al., 1983; Molyneux et al., 1989; Agbenyega et al., 2000). Similar observations have been made concerning canine babesiosis (Button, 1979; Leisewitz et al., 2001; Nel et al., 2004; Jacobson and Lobetti, 2005). Children and pregnant women with falciparum malaria are at greater risk of developing hypoglycaemia (Looareesuwan et al., 1985; White et al., 1987; Davis et al., 1994; Agbenyega et al., 2000; Dzeing-Ella et al., 2005). Hypoglycaemia is encountered in 20-30\% of children with cerebral malaria (Newton and Krishna, 1998), and was found in 5 out of 10 pregnant woman with cerebral malaria in a watershed study conducted in Thailand in 1983 (White et al., 1983). The similarities between canine babesiosis and human malaria are striking (Maegraith et al., 1957; Welzl et al., 2001). These diseases share numerous clinical and pathophysiological characteristics, as do human babesiosis and malaria (Reyers et al., 1998; Clark and Jacobson, 1998). Haemolysis, severe systemic inflammation and associated pro-inflammatory cytokine production are thought to contribute to disease pathogenesis in both babesiosis and malaria (Reyers et al., 1998).

Inappropriately high plasma insulin concentrations were found in association with the hypoglycaemic episodes seen in many human patients with falciparum malaria (notably in children and pregnant women), a phenomenon attributed to quinine-induced insulin secretion (White et al., 1983; Krishna and White, 1996; Agbenyega et al., 2000). Hyperinsulinaemia has, however, been identified in cases of malaria prior to quinine administration (Looareesuwan et al., 1985; Taylor et al., 1988; Shalev et al., 1992). In addition, hyperinsulinaemia, in conjunction with hypoglycaemia, is a frequent occurrence in experimental murine models of malaria (Elased and Playfair, 1994), where insulin concentrations as high as $30,960 \mathrm{pmol} / \mathrm{L}$ were obtained.

The authors of the above study concluded that a subgroup of human malaria patients may exist where hyperinsulinaemia is the cause of hypoglycaemia. A similar phenomenon of inappropriate insulin secretion, resulting in concurrent hyperinsulinaemia and hypoglycaemia, may exist in canine babesiosis. Little is known regarding specific endocrine changes in dogs with babesiosis. Schoeman et al. (2007) showed that high basal serum cortisol and ACTH concentrations at presentation were associated with increased mortality in dogs suffering 
from B. canis rossi infection. Plasma insulin concentration, and its relationship with abnormal blood glucose concentrations, has not been studied in canine babesiosis. The purpose of this study was to quantify the plasma insulin concentrations of dogs suffering from babesiosis in an attempt to identify a putative association between hyperinsulinaemia and hypoglycaemia.

\section{Materials and methods}

This prospective, cross-sectional study included 98 dogs presented to the Outpatients Clinic of the Onderstepoort Veterinary Academic Hospital (OVAH) with naturally occurring virulent babesiosis. Typical clinical signs (Jacobson, 2006) and microscopic identification of large Babesia spp. parasites on thin peripheral blood smears stained with Kyro-QuickTM stain (Kyron Laboratories, Benmore, South Africa) were considered diagnostic.

Study design - the individual ages and bodyweights of dogs were recorded at presentation, and the owners completed a questionnaire documenting the duration of illness and time since last meal. Clinical parameters including habitus, temperature, pulse, respiratory rate, presence of icterus, and neurological status (i.e. collapse, coma, seizures) were noted. Informed owner consent was obtained and pre-treatment blood samples were collected into pre-cooled ethylenediamine tetraacetic acid (EDTA) and sodium fluoride-anticoagulated plastic tubes (Vacutainer ${ }^{\mathrm{TM}}$, BD Vacutainer Systems, Plymouth, UK) at the time of presentation, and kept on ice until processing. Samples were centrifuged at $48 \mathrm{C}$ for $10 \mathrm{~min}$ within $1 \mathrm{~h}$ of collection. Plasma was separated and initially stored at

$18^{\circ} \mathrm{C}$. Fluorideanticoagulated samples were submitted for glucose determination (storage time 0-3 days) using the hexokinase method (Sonnenwirth and Jarett, 1980; Kaplan and Pesce, 1984). EDTA-anticoagulated plasma samples were transferred to a $80^{\circ} \mathrm{C}$ freezer and batched for subsequent insulin determination. Plasma insulin concentration was determined using a commercially available solid-phase radioimmunoassay kit (Coat-A-Count ${ }^{\circledR}$, DPC, Los Angeles, CA) previously validated for use in dogs (Kaplan and Pesce, 1984; Parsons et al., 2002). All samples were screened for Ehrlichia canis and $B$. canis vogeli using polymerase chain reaction (PCR) and reverse line blot (RLB) as previously described (Matjila et al., 2004). PCR was conducted with a set of primers that amplified a 460-540 base pair fragment of the 18S SSU rRNA spanning the V4 region conserved for Babesia and Theileria. The Ehrlichia PCR amplified the V1 hypervariable region of the 16S SSU rRNA (Schouls et al., 1999; Bekker et al., 2002). The membrane used for RLB-included probes for B. canis vogeli, B. canis rossi, B. c. canis and Ehrlichia canis. The study was reviewed and approved by the institutional Animal Use and Care Committee. Normoglycaemia was defined as blood glucose concentrations in the range 3.3-5.5 mmol/L. Glucose values in the hypoglycaemic group included values below $3.3 \mathrm{mmol} / \mathrm{L}$ (Turnwald and Troy, 1983; Walters and Drobatz, 1992), and hyperglycaemia was defined as blood glucose concentration $>5.5 \mathrm{mmol} / \mathrm{L}$. The normal range for canine plasma insulin concentration is 35-180 pmol/L (Reimers et al., 1982; Parsons et al., 2002).Values below10.7 $\mathrm{pmol} / \mathrm{L}$ were considered to be belowthe limits of detection of the assay (Parsons et al., 2002), and were entered in the statistical analysis as $10.7 \mathrm{pmol} / \mathrm{L}$. Animals with a plasma insulin concentration above $180 \mathrm{pmol} / \mathrm{L}$ were considered to be hyperinsulinaemic.

Statistical analysis-parameters were tested for normal distribution using the one-sample Kolmogorov- Smirnov test. Differences in the median values of the variables in the three glucose groups were analyzed for nonparametric data with the Kruskal-Wallis test and subsequently using the Mann-Whitney U-test for pairwise comparisons. Normally distributed data were analyzed using one-way ANOVA, with the Bonferroni correction for multiple comparisons. For all comparisons, differences were considered significant when $p<0.05$. Values for nonparametric data in the text are given as median and interquartile range (IQR) and for parametric data as mean and range. Statistical analysis was performed using a commercial software package (SPSS 14.0, 2005, SPSS Inc., Chicago, IL).

\section{Results}

Ninety-eight dogs presenting successively to the OVAH were sampled. All dogs included in the study had large babesia parasites present on their blood smear. A probe for the small babesia, Babesia gibsoni, was not included in the RLB, and thus co-infection with this parasite could not be ruled out. PCR produced three positive results for $B$. canis vogeli, resulting in the exclusion of these cases from the study. The results of one dog were also censored due to prior intravenous dextrose administration. Thus the data from 94 dogs were included in the statistical analysis. Median age for all dogs was 16 months (IQR 9-39 months). Body temperature was the only normally distributed variable and the mean body temperature was $39.1^{\circ} \mathrm{C}$ (range $32.7-41.1^{\circ} \mathrm{C}$ ). The median pulse rate was 130 beats per minute (IQR 112-150). The median respiratory rate was 46 breaths per minute (IQR 33-60). The median number of days ill prior to presentation was 2 days (IQR 2-4 days), and the median time since last meal was $24 \mathrm{~h}$ (IQR 12-48 h, data available for 87 dogs). Seventeen dogs (18\%) were icteric, and 14 dogs (15\%) demonstrated neurological signs, including collapse (11/14) and coma (3/14). Animals were retrospectively assigned to one of the three groups according to their blood glucose concentration at presentation: hypoglycaemic ([glucose] < $3.3 \mathrm{mmol} / \mathrm{L} ; n=16$; range 1.0-3.2 mmol/L), normoglycaemic ([glucose] 3.3-5.5 mmol/L; $n=62 ; \mathrm{range}$ 3.4-5.5 mmol/L), and hyperglycaemic ([glucose] > $5.5 \mathrm{mmol} / \mathrm{L} ; n=16$; range 5.6-6.6 mmol/L). The median insulin concentrations for the three groups were $10.7 \mathrm{pmol} / \mathrm{L}$ (10.7-18.8 pmol/L), $10.7 \mathrm{pmol} / \mathrm{L}$ (10.7-29.53 pmol/L), and $21.7 \mathrm{pmol} / \mathrm{L}$ (10.7-45.74 pmol/L), respectively (Fig. 1). The median insulin concentrations for the hypoglycaemic and normoglycaemic groups were below the detection limit of the assay. Although there was a trend for insulin concentration to increase as blood glucose concentration increased, no significant difference in insulin concentration was found between the three groups $\left(x^{2} k-w=1.972, p=0.373\right)$. However, two dogs were found to have insulin concentrations above the reference range. One dog was mildly hyperinsulinaemic 
(case number 56; insulin concentration $198 \mathrm{pmol} / \mathrm{L}$ ); the other was severely hyperinsulinaemic (case number 34; insulin concentration $1563 \mathrm{pmol} / \mathrm{L}$ ). Of the 16 hypoglycaemic dogs, 6 had detectable plasma insulin concentrations (Table 1). Excluding case number 34, the range of insulin concentrations in the remaining five hypoglycaemic dogs was $18.45-32.6 \mathrm{pmol} / \mathrm{L}$.

Median duration of illness, time since last meal, pulse and respiration rate did not differ between the groups. Hypoglycaemic dogs (median 13.5 months) were significantly younger than normoglycaemic dogs (median 24 months) ( $p=0.04)$. Hyperglycaemic dogs (median 9 months) were also significantly younger than normoglycaemic dogs $(p<0.001$ ). Both hypoglycaemic dogs (median $5.6 \mathrm{~kg}$ ) and hyperglycaemic dogs (median $9 \mathrm{~kg})$ had significantly lower bodyweight than normoglycaemic dogs (median $20 \mathrm{~kg})(p<0.01$ for both). Hypoglycaemic dogs (mean $37.7^{\circ} \mathrm{C}$; range 33-40.2) had significantly lower body temperature than normoglycaemic dogs (mean 39.6 ${ }^{\circ} \mathrm{C}$; range 33-41.1) $(p<0.001)$, but did not differ significantly from hyperglycaemic dogs (mean $38.8^{\circ} \mathrm{C}$; range $32.7-40.4)(p=0.077)$

\section{Discussion}

In the dogs sampled the median insulin concentration in the hypoglycaemic and normoglycaemic groups was below the detection limit of the radioimmunoassay, whereas the median insulin concentration in the hyperglycaemic group was within the upper part of the reference range. An apparent trend for insulin concentration to increase as blood glucose concentration increased was identified. These results suggest that insulin secretion was inhibited in the hypoglycaemic and normoglycaemic dogs, and that an appropriate physiological relationship exists between glucose and insulin in this group of dogs with babesiosis. Six of the hypoglycaemic dogs did, however, have detectable plasma insulin concentrations. Hyperinsulinaemia was identified in two cases, and was present in only one dog (1\%) in conjunction with hypoglycaemia. The first of the hyperinsulinaemic dogs, a 6-yearold intact male Fox Terrier (insulin concentration = $198 \mathrm{pmol} / \mathrm{L}$; glucose concentration $=4.8 \mathrm{mmol} / \mathrm{L}$ ), had a plasma insulin concentration marginally above the reference range. The dog was suffering from complicated babesiosis, was comatose and severely icteric, and died shortly after presentation. Considering that the dog was comatose at presentation it is unlikely to have eaten in the hours immediately before blood sampling, and postprandial insulin release is therefore unlikely. Peripheral insulin resistance commonly coexists with hyperinsulinaemia in cases of human malaria (Davis et al., 1990; van Thien et al., 2006), and may account for the normal blood glucose concentration associated with hyperinsulinaemia in this dog. Pancreatic b cell neoplasia could not be ruled out in this case. In the second dog with hyperinsulinaemia, a 2-year-old male Boxer (insulin concentration = $1653 \mathrm{pmol} / \mathrm{L}$; glucose concentration $=2.9 \mathrm{mmol} / \mathrm{L}$ ), the insulin concentration was dramatically higher than normal physiological values, and is higher even than values commonly encountered in dogs with b-cell neoplasia (Dunn et al., 1992). This dog was also moderately hypoglycaemic, and was collapsed despite a haematocrit of $27 \%$. This patient recovered and was discharged, and represents the only case identified in this study with concurrent hypoglycaemia and hyperinsulinaemia. The age of this dog, in addition to the absence of clinical signs of episodic weakness or collapse over an 18-month follow-up period, suggest that pancreatic b-cell neoplasia as a source of the insulin is unlikely. It is not known whether high parasitaemia might be linked to hyperinsulinaemia. Hypoglycaemia and collapse have been linked to high parasitaemia in babesiosis (Jacobson and Lobetti, 2005; Böhm et al., 2006) and malaria (White et al., 1983). A small sub-population of dogs suffering from babesiosis may therefore exist in which inappropriate insulin secretion occurs, increasing the risk for hypoglycaemia.

It has been suggested that a plasma insulin concentration greater than $72 \mathrm{pmol} / \mathrm{L}$, with a concurrent glucose concentration less than $2.8 \mathrm{mmol} / \mathrm{L}$, constitutes an inappropriate excess of insulin (Feldman and Nelson, 2004). Although six of the hypoglycaemic dogs had detectable insulin in their plasma, their values were at the lower end of the normal range. Only one hypoglycaemic dog had insulin concentrations greater than 72 pmol/L (the severely hyperinsulinaemic case discussed in the previous paragraph).

The results of this study confirm that, for the most part, insulin concentrations in dogs with babesiosis are low, which is similar in people with severe malaria (Looareesuwan et al., 1985; White, 2003; van Thien et al., 2006). Low insulin concentrations can therefore be considered a normal finding in the ill, anorexic dogs studied here. Fasting plays a major role in the suppression of insulin secretion. In healthy dogs, fasting results in mild hypoglycaemia with moderate decreases in insulin concentration (de Bruijne et al., 1981). Dogs suffering from babesiosis are usually anorexic as a result of their illness, and can be expected to have appropriately low plasma insulin concentrations. When blood glucose concentrations fall further (below $2.8 \mathrm{mmol} / \mathrm{L}$ ), insulin secretion is completely inhibited (de Bruijne et al., 1981; Leifer and Peterson, 1984;Walters and Drobatz, 1992; Feldman and Nelson, 2004). Insulin should therefore be practically undetectable in the plasma of hypoglycaemic patients, due to a lack of direct b-cell stimulation, and an increase in a adrenergic inhibition of pancreatic insulin secretion (Feldman and Nelson, 2004).

Hypoglycaemia was present in 16 of 94 (17\%) dogs included in this study. A proportion of these dogs showed signs consistent with neuroglycopoenia, including collapse and coma. Five of the 16 dogs with hypoglycaemia were icteric, a finding similar to those of a previous study (Keller et al., 2004), where icterus was established as a significant risk factor for the development of hypoglycaemia. Hypoglycaemic dogs were significantly younger, with lower body weight (probably a function of younger age) than normoglycaemic dogs. In a previous study, dogs less than 6 months of age were 2.8 times more likely to develop hypoglycaemia (Keller et al., 2004). The majority $(95.7 \%)$ of hypoglycaemic dogs in that study were hospitalised due to the presence of severe disease. In humans, children commonly present with hypoglycaemia in conjunction with a variety of severe diseases (Kawo et al., 1990; 
Solomon et al., 1994; Osier et al., 2003), despite having fully developed gluconeogenic capacity at birth (Bier et al., 1977), suggesting that glycogen stores may be limited. This may apply to young dogs, where lower levels of stored glycogen may reduce the host's ability to withstand the physiologic stresses of severe disease, and such animals cannot adequately compensate during periods of increased demand for carbohydrate fuels. In human patients with trauma and sepsis, a significant increase in glucose consumption is mediated by release of inflammatory cytokines such as tumour necrosis factor from the macrophage-rich spleen, liver and lungs (Mizock, 1995). In human malaria, a disease pathophysiologically similar to canine babesiosis, increased anaerobic glycolysis due to microvascular obstruction by sequestrated parasitized red blood cells, parasite demands for glucose, decreased hepatic blood flow, compromised hepatic function, and failure of gluconeogenesis may contribute to the pathogenesis of hypoglycaemia (White, 2003; Jacobson and Lobetti, 2005). Similar mechanisms are likely to contribute to the development of hypoglycaemia in dogs with severe canine babesiosis. Due to the relatively small size of the hypoglycaemic group $(n=16)$, the incidence of insulin-induced hypoglycaemia in canine babesiosis may have been underestimated by this study.

Further sampling of greater numbers of hypoglycaemic dogs may identify more dogs with hyperinsulinaemia. This study was cross-sectional, and did not follow temporal patterns in blood glucose and insulin concentrations. A more detailed understanding of fluctuations in the glucose: insulin relationship during the natural history of this disease might be gained if these parameters were to be measured in dogs not only at presentation, but also at various points following admission.

\section{Conclusion}

We conclude that hyperinsulinaemia is an infrequent cause of hypoglycaemia in dogs with babesiosis attributable to infection with $B$. canis rossi. A small sub-population of dogs may exist in which significant hyperinsulinaemia could result in clinical hypoglycaemia. Future studies involving larger numbers of hypoglycaemic dogs may assist in quantifying this phenomenon.

\section{Acknowledgements}

The authors would like to express their gratitude to the following: Mesdames Lea Goddard, Annemarie Human, Elsbe Myburgh, Gertie Pretorius, Cheryl Booth and Desire' Rees for laboratory assistance; final-year veterinary students and colleagues for notifying the authors of subjects for the study and helping with the handling of the dogs; PCR laboratory at the Department of Veterinary Tropical Diseases, University of Pretoria. This study was financially supported by the Jowett fund, University of Cambridge and the research fund of the Department of Companion Animal Clinical Studies, University of Pretoria.

\section{References}

1. Agbenyega, T., Angus, B.J., Bedu-Addo, G., Baffoe-Bonnie, B., Guyton, T., Stacpoole, P.W., Krishna, S., 2000. Glucose and lactate kinetics in children with severe malaria. J. Clin. Endocr. Metab. 85, 1569-1576.

2. Bakker, J., Gris, P., Coffernils, M., Kahn, R.J., Vincent, J.L., 1996. Serial blood lactate levels can predict the development of multiple organ failure following septic shock. Am. J. Surg. 171, 221-226.

3. Bekker, C.P., de Vos, S., Taoufik, A., Sparagano, O.A., Jongejan, F., 2002. Simultaneous detection of Anaplasma and Ehrlichia species in ruminants and detection of Ehrlichia ruminantium in Amblyomma variegatum ticks by reverse line blot hybridization. Vet. Microbiol. 89, 223-238.

4. Bier, D.M., Leake, R.D., Haymond, M.W., Arnold, K.J., Gruenke, L.D., Sperling, M.A., Kipnis, D.M., 1977. Measurement of "true" glucose production rates in infancy and childhood with 6,6- dideuteroglucose. Diabetes 26, 1016-1023.

5. Böhm, M., Leisewitz, A.L., Thompson, P.N., Schoeman, J.P., 2006. Capillary and venous Babesia canis rossi parasitaemias and their association with outcome of infection and circulatory compromise. Vet. Parasitol. 141, 18-29.

6. Button, C., 1979. Metabolic and electrolyte disturbances in acute canine babesiosis. J. Am. Vet. Med. Assoc. 175, 475-479.

7. Clark, I.A., Jacobson, L.S., 1998. Do babesiosis and malaria share a common disease process? Ann. Trop. Med. Parasitol. 92, 483-488.

8. Davis, T.M., Pukrittayakamee, S., Supanaranond, W., Looareesuwan, S., Krishna, S., Nagachinta, B., Turner, R.C., White, N.J., 1990. Glucose metabolism in quinine-treated patients with uncomplicated falciparum malaria. Clin. Endocrinol. 33, 739-749.

9. Davis, T.M., Suputtamongkol, Y., Spencer, J.L., Wilson, S.G., Mekhton, S., Croft, K.D., White, N.J., 1994. Glucose turnover in pregnant women with acute malaria. Clin. Sci. 86, 83-90.

10. de Bruijne, J.J., Altszuler, N., Hampshire, J., Visser, T.J., Hackeng, W.H.L., 1981. Fat mobilization and plasma hormone levels in fasted dogs. Metabolism 30, 190-194.

11. Dunn, J.K., Heath, M.F., Herrtage, M.E., Jackson, K.F., Walker, M.J., 1992. Diagnosis of insulinoma in the dog: a study of 11 cases. J. Small Anim. Pract. 33, 514-520.

12. Dzeing-Ella, A., Nze Obiang, P.C., Tchoua, R., Planche, T., Mboza, B., Mbounja, M., Muller-Roemer, U., Jarvis, J., Kendjo, E., Ngou-Milama, E., Kremsner, P.G., Krishna, S., Kombila, M., 2005. Severe falciparum malaria in Gabonese children: clinical and laboratory features. Malaria J. 4, 1. Elased, K., Playfair, J.H.L., 
1994. Hypoglycemia and hyperinsulinemia in rodent models of severe malaria infection. Infect. Immun. 62 , 5157-5160.

13. Feldman, E.C., Nelson, R.W., 2004. Beta-cell neoplasia: insulinoma. In: Feldman, E.C., Nelson, R.W. (Eds.), Canine and Feline Endocrinology and Reproduction. Elsevier Science, St. Louis, pp. 616-644.

14. Jacobson, L.S., 2006. The South African form of severe and complicated canine babesiosis: clinical advances 1994-2004. Vet. Parasitol. 138, 126-139.

15. Jacobson, L.S., Lobetti, R.G., 2005. Glucose, lactate and pyruvate concentrations in dogs with babesiosis. Am. J. Vet. Res. 66, 244-250.

16. Kaplan, L.A., Pesce, A.J., 1984. Clinical chemistry: Theory, Analysis and Correlation. Mosby Company, St Louis. Kawo, N.G., Msengi, A.E., Swai, A.B.M., Chuwa, L.M., Alberti, K.G., McLarty, D.G., 1990. Specificity of hypoglycaemia for cerebral malaria in children. Lancet 336, 454-457.

17. Keller, N., Jacobson, L.S., Nel, M., de Clerq, M., Thompson, P.N., Schoeman, J.P., 2004. Prevalence and risk factors of hypoglycaemia in virulent canine babesiosis. J. Vet. Intern. Med. 18, 265-270.

18. Krishna, S., White, N.J., 1996. Pharmacokinetics of quinine, chloroquine and amodiaquine. Clinical implications. Clin. Pharmacokinet. 30, 263-299.

19. Leifer, C.E., Peterson, M.E., 1984. Hypoglycemia. Vet. Clin. N. Am. Small 14, 873-889.

20. Leisewitz, A.L., Jacobson, L.S., de Morais, H.S., Reyers, F., 2001. The mixed acid-base disturbances of severe canine babesiosis. J. Vet. Intern. Med. 15, 445-452.

21. Lobetti, R.G., Jacobson, L.S., 2001. Renal involvement in dogs with babesiosis. J. S. Afr. Vet. Assoc. 72, 23-28.

22. Looareesuwan, S., Phillips, R.E., White, N.J., Kietinun, S., Karbwang, J., Rackow, C., Turner, R.C., Warrell, D.A., 1985. Quinine and severe falciparum malaria in late pregnancy. Lancet 2, 4-8.

23. Maegraith, B., Gilles, H.M., Devakul, K., 1957. Pathological processes in Babesia canis infections. Z. Tropenmed. Parasitol. 8, 485-514.

24. Matjila, P.T., Penzhorn, B.L., Bekker, C.P., Nijhof, A.M., Jongejan, F., 2004. Confirmation of occurrence of Babesia canis vogeli in domestic dogs in South Africa. Vet. Parasitol. 122, 119-125.

25. Mizock, B.A., 1995. Alterations in carbohydrate metabolism during stress: a review of the literature. Am. J. Med. 98, 75-84.

26. Mohr, A.J., Lobetti, R.G., van der Lugt, J.J., 2000. Acute pancreatitis: a newly recognised potential complication of canine babesiosis. J. S. Afr. Vet. Assoc. 71, 232-239.

27. Molyneux, M.E., Taylor, T.E., Wirima, J.J., Borgstein, A., 1989. Clinical features and prognostic indicators in paediatric cerebral malaria: a study of 131 comatose Malawian children. Quat. J. Med. 71, 441-459.

28. Nel, M., Lobetti, R.G., Keller, N., Thompson, P.N., 2004. Prognostic value of blood lactate, blood glucose, and hematocrit in canine babesiosis. J. Vet. Intern. Med. 18, 471-476.

29. Newton, C.R., Krishna, S., 1998. Severe falciparum malaria in children: current understanding of pathophysiology and supportive treatment. Pharmacol. Ther. 79, 1-53.

30. Osier, F.H., Berkley, J.A., Ross, A., Sanderson, F., Mohammed, S., Newton, C.R., 2003. Abnormal blood glucose concentrations on admission to a rural Kenyan district hospital: prevalence and outcome. Arch. Dis. Child 88, 621-625.

31. Parsons, S.E., Drobatz, K.J., Lamb, S.V.,Ward, C.R., Hess, R.S., 2002. Endogenous serum insulin concentration in dogs with diabetic ketoacidosis. J. Vet. Emerg. Crit. Care 12, 147-152.

32. Reimers, T.J., Cowan, R.G., McCann, J.P., Ross, M.W., 1982. Validation of a rapid solid-phase radioimmunoassay for canine, bovine and equine insulin. Am. J. Vet. Res. 43, 1274-1278.

33. Reyers, F., Leisewitz, A.L., Lobetti, R.G., Milner, R.J., Jacobson, L.S., van Zyl, M., 1998. Canine babesiosis in South Africa: more than one disease. Does this serve as a model for falciparum malaria? Ann. Trop. Med. Parasitol. 92, 503-511.

34. Schoeman, J.P., Rees, P., Herrtage, M.E., 2007. Endocrine predictors of mortality in canine babesiosis caused by Babesia canis rossi. Vet. Parasitol. 148, 75-82.

35. Schouls, L.M., van de Pol, I., Rijpkema, S.G., Schot, C.S., 1999. Detection and identification of Ehrlichia, Borrelia burgdorferi sensu lato, and Bartonella species in Dutch Ixodes ricinus ticks. J. Clin. Microbiol. 37, 2215-2222.

36. Shalev, O., Tsur, A., Rahav, G., 1992. Falciparum malaria-induced hypoglycaemia in a diabetic patient. Postgrad. Med. J. 68, 281-282.

37. Solomon, T., Felix, J.M., Samuel, M., Dengo, G.A., Saldanha, R.A., Schapira, A., Phillips, R.E., 1994. Hypoglycaemia in paediatric admissions in Mozambique. Lancet 343, 149-150.

38. Sonnenwirth, A.C., Jarett, L., 1980. Gradwohl's Clinical Laboratory Methods and Diagnosis. Mosby Company, St Louis.

39. Taylor, T.E., Molyneux, M.E.,Wirima, J.J., Fletcher, K.A., Morris, K., 1988. Blood glucose levels in Malawian children before and during the administration of intravenous quinine for severe falciparum malaria. N. Engl. J. Med. 319, 1040-1047.

40. Turnwald, G.H., Troy, G.C., 1983. Hypoglycemia. Part I. Carbohydrate metabolism and laboratory evaluation. Comp. Cont. Educ. Small Anim. Pract. 5, 932-937.

41. van Thien, H.V., Kager, P.A., Sauerwein, H.P., 2006. Hypoglycemia in falciparum malaria: is fasting an unrecognized and insufficiently emphasized risk factor? Trends Parasitol. 22, 410-415.

42. Walters, P.C., Drobatz, K.J., 1992. Hypoglycemia. Comp. Cont. Educ. Pract. 14, 1150-1158. 
43. Welzl, C., Leisewitz, A.L., Jacobson, L.S., Vaughan-Scott, T., Myburgh, E., 2001. Systemic inflammatory response syndrome and multiple-organ damage/dysfunction in complicated canine babesiosis. J. S. Afr. Vet. Assoc. 72, 158-162.

44. White, N.J., 2003. Malaria. In: Cook, G.C., Zumla, A.I. (Eds.), Manson's Tropical Diseases. W.B. Saunders, St Louis, pp. 1205-1295.

45. White, N.J., Warrell, D.A., Chanthavanich, P., Looareesuwan, S., Warrell, M.J., Krishna, S., Williamson, D.H., Turner, R.C., 1983. Severe hypoglycemia and hyperinsulinemia in falciparum malaria. N. Engl. J. Med. 309, 61-66.

46. White, N.J., Miller, K.D., Marsh, K., Berry, C.D., Turner, R.C., Williamson, D.H., Brown, J., 1987. Hypoglycaemia in African children with severe malaria. Lancet 1, 708-711.

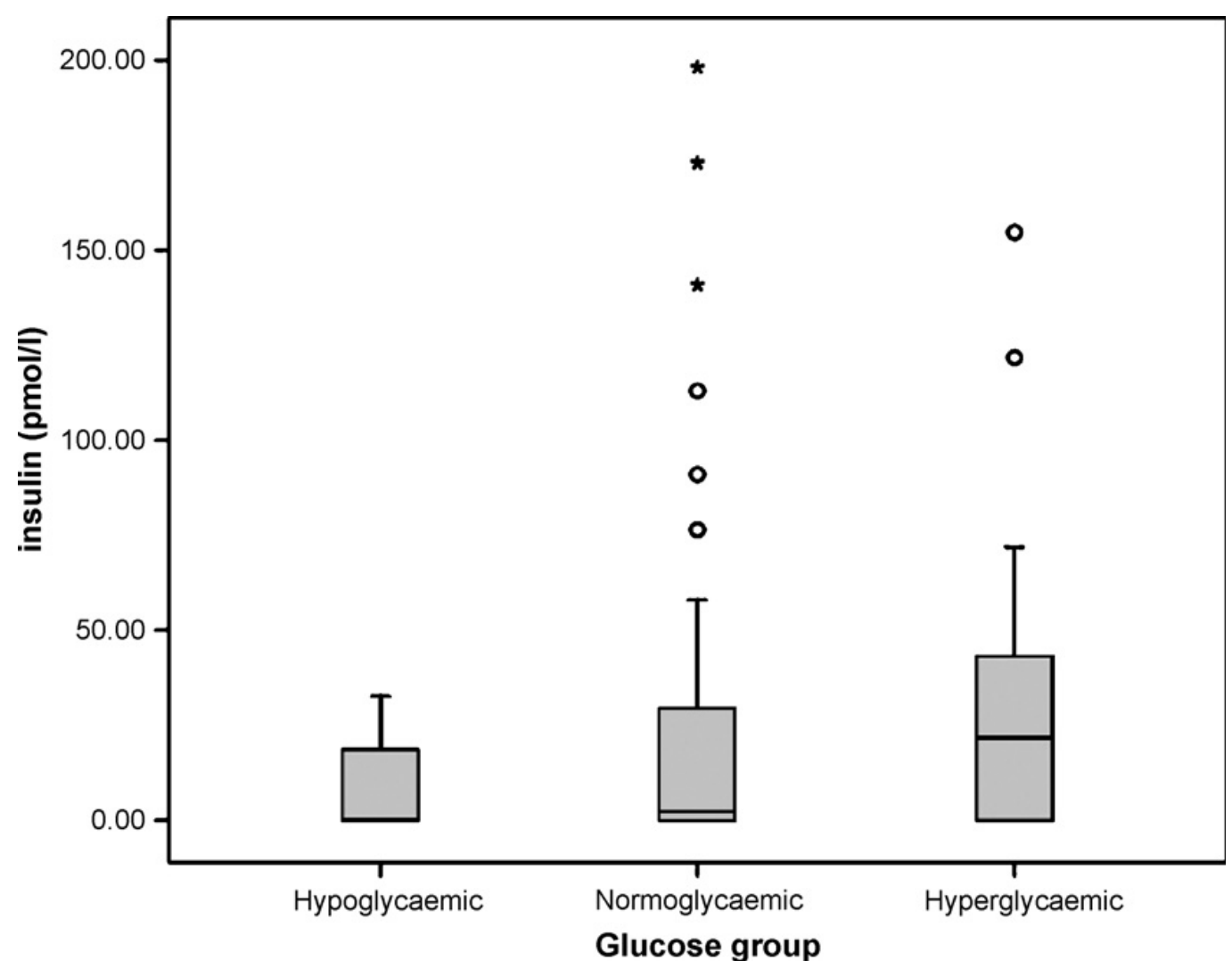

Fig. 1. Boxplot showing insulin concentrations for the three blood glucose concentration groups. The box represents the interquartile range, the median is shown as a horizontal bar, and the $T$ bars represent the main body of data. Outliers are indicated as open circles and stars, and the severely hyperinsulinaemic case (insulin concentration $1653 \mathrm{pmol} / \mathrm{L}$ ) is not shown

\section{Table 1}

Table showing glucose and insulin concentrations for the 16 hypoglycaemic dogs with babesiosis

\begin{tabular}{|lll|}
\hline Case number & $\begin{array}{l}\text { Glucose concentration } \\
(\mathrm{mmol} / \mathrm{L})\end{array}$ & $\begin{array}{l}\text { Insulin concentration } \\
(\mathrm{pmol} / \mathrm{L})\end{array}$ \\
\hline 5 & 2.4 & 10.7 \\
18 & 2.2 & 18.45 \\
26 & 3.0 & 10.7 \\
31 & 1.3 & 10.7 \\
34 & 2.9 & 1653 \\
37 & 2.5 & 10.7 \\
44 & 1.4 & 10.7 \\
46 & 2.1 & 10.7 \\
55 & 2.4 & 18.81 \\
57 & 3.2 & 18.74 \\
61 & 1.1 & 28.29 \\
62 & 2.2 & 32.6 \\
79 & 2.0 & 10.7 \\
84 & 2.8 & 10.7 \\
88 & 1.6 & 10.7 \\
90 & 1.0 & 10.7 \\
& & \\
\hline
\end{tabular}

Values recorded as 10.7 refer to insulin concentrations below the detection limit of the assay. 